\title{
Evaluation of the effects of radiotherapy as a mode of treatment in management of advanced cancer cervix
}

\author{
Rajeshwari BV*
}

Department of Obstetrics and Gynecology, Kamineni Institute of Medical Sciences, Nalgonda, Telangana, India

Received: 11 December 2016

Accepted: 17 December 2016

\section{*Correspondence:}

Dr. Rajeshwari BV.,

E-mail: dr.rajeshwari.obg@gmail.com

Copyright: (c) the author(s), publisher and licensee Medip Academy. This is an open-access article distributed under the terms of the Creative Commons Attribution Non-Commercial License, which permits unrestricted non-commercial use, distribution, and reproduction in any medium, provided the original work is properly cited.

\section{ABSTRACT}

Background: Throughout world over 500000 new cases of invasive cancer cervix are detected each year and account for $15 \%$ of new cancer and 200000 deaths annually. In India more than 90,000 women suffer annually from cervical cancer and mortality of them report in advanced stage of disease.

Methods: All cases were investigated with routine hematological and biochemical examination, $\mathrm{x}$-ray chest, and ultrasonography of the abdomen and pelvis before starting radiotherapy treatment. All patients were examined and staged clinically according to the International Federation of gynaecologists and Oncologists (FIGO) staging system.

Results: Majority of the patients $50 \%$ were in stage III B, followed by stage III A about $21 \%$ and $20 \%$ in stage IIB. During follow up, in study group maximum number of patients about $76 \%$ presented with skin changes like dryness, erythema, discoloration, diarrhea in $40 \%$, obstructive uropathy in $16 \%$, and in $4 \%$ developed vaginal stenosis.

Conclusions: Today, malignancy is the challenge not only to the gynecologist, radiation oncologist but also to the patient. But because of newer technique and advances in medical field update in infrastructure gives to the specialist new dimension to treat the patient as well as raise of hopes to the patient.

Keywords: Cancer Cervix, Chemotherapy, Radiotherapy

\section{INTRODUCTION}

"Success with cancer (patient) treatment is not survival at any cost but, rather to improve the quality of life for the patient" Carcinoma cervix is a treatable disease but unfortunately not yet prevented!! ${ }^{1}$

It is one of the most common malignancy in females ${ }^{2}$ and a major public health problem in India and second most common female cancer in the developed countries.

It is a preventable disease if detected in early stages. In India, cervical cancer is the one of the cause of death among between the ages 20 and 40 years. ${ }^{3}$

Throughout world over 500000 new cases of invasive cancer cervix are detected each year and account for $15 \%$ of new cancer and 200000 deaths annually. ${ }^{4}$
In India more than 90,000 women suffer annually from cervical cancer and mortality of them report in advanced stage of disease.

Because of easy accessibility of cervix for direct visual inspection and cytological evaluation, it has a high cure rate when detected in early stage, however because the females in developing countries are ignorant about their health leading to late diagnosis and presentation with advanced clinical stages.

The management of advanced carcinoma cervix changed soon after the discovery of Roentgen x-ray in 1895 and Radium in 1898. Margaret cleaves of New York City used radium for the first time for the treatment of ca cervix. ${ }^{5}$ Radiotherapy has shown a good success rate than surgery and hence remained the mode of treatment in late, advanced inoperable stages. 
As most of the cases present at advanced stages, such as stage III and IV, in which surgery is not possible, radiotherapy plays an important role in these patients. ${ }^{6}$

Radiation has been used successfully to treat cervical cancer for nearly a century. The combination of external beam irradiation and brachytherapy has been shown to be an effective treatment for Carcinoma Cervix patients. The success of brachytherapy requires delivery of a high radiation dose to the tumor while sparing, to some degree, the surrounding normal tissues.

\section{METHODS}

100 patients of advanced cancer cervix registered at oncology OPD/Ward were selected for the study. Out of the 100 patients, 50 cases who received combined chemotherapy and radiotherapy were taken as study group and 50 cases who received only radiotherapy were labelled as control group. They were analysed prospectively for residual disease, local recurrences, and distant metastases, effects of chemo radiation and disease free survival.

All were belonging to FIGO stage II B to IV A, aged between 30- 80 years, with histologically proven cancer cervix. With stage 0 to II A, stage IV B with distant metastases and who were medically unfit were excluded.

\section{Inclusion criteria}

- Advanced cancer cervix registered at oncology OPD/Ward

- $\quad$ Age group of $30-80$ years

\section{Exclusion criteria}

- Seriously ill patients

- Not willing to participate in the study

All cases were investigated with routine hematological and biochemical examination, $\mathrm{x}$-ray chest, and ultrasonography of the abdomen and pelvis before starting radiotherapy treatment. All patients were examined and staged clinically according to the International Federation of gynaecologists and Oncologists (FIGO) staging system. Histopathological confirmation was done for all patients Pre-treatment hemoglobin level was though not taken into consideration for survival analysis, but was kept above $10 \mathrm{gm} \%$, by giving blood/packed red cell transfusion.

Our patients were treated with external radiation of 5000$6000 \mathrm{~Gy}$ in the dose of $200 \mathrm{~Gy} /$ day alternately in the anterior and posterior pelvis in 25-30 settings. This was followed by intracavitary radiation or brachytherapy of $1200-1500$ Gy given in two to four sittings. It consists of one tandem in the uterine cavity and two ovoids in the vagina.
In study group, along with radiotherapy, chemotherapy was given. The choice of chemotherapy was weekly cisplatin $40 \mathrm{mg} / \mathrm{m}^{2}$. Chemotherapy act as a radiosensitizer and it interacts synergistically with radiotherapy. Cell cycle synchronization, inhibition of repair of sub lethal damage or inhibition of recovery from potential lethal radiation damage and hypoxic cell sensitization are some of the possible mechanisms of chemotherapy radiotherapy interaction.

Patients were followed up for a period of 2 years for morbidity, mortality, recurrences and other complications related chemo radiation.

In the first year of complete treatment patients were called every 3 months and afterword's every 6 months, general examination, per speculum, per vaginal examination, Pap smear, USG, CT Scan(if required) done to detect clinical response or recurrence.

Loco regional response was assessed in terms of complete response, partial response, No response and progressive disease

\section{RESULTS}

Table 1: Age incidence.

\begin{tabular}{|lll|}
\hline Age $($ yrs $)$ & CT+RT & RT \\
\hline $31-40$ & 3 & 2 \\
\hline $41-50$ & 24 & 16 \\
\hline $51-60$ & 23 & 6 \\
\hline $61-70$ & - & 20 \\
\hline $71-80$ & - & 6 \\
\hline Total & 50 & 50 \\
\hline
\end{tabular}

Chi square $=8.26 ; \mathrm{df}=2 ; \mathrm{P}<0.05$

Table 1 show that the 47 patients in 41-60 years receiving chemo radiotherapy and 26 patients between $60-80$ years receiving radiotherapy, showing that age acts as an independent risk factor for advanced stage of cancer cervix.

Table 2: Stage wise distribution.

\begin{tabular}{|lll|}
\hline Stage & CT+RT & RT \\
\hline II B & 12 & 8 \\
\hline III A & 10 & 11 \\
\hline III B & 25 & 25 \\
\hline IVA & 3 & 6 \\
\hline Total & 50 & 50 \\
\hline
\end{tabular}

Majority of the patients $50 \%$ were in stage III B, followed by stage III A about $21 \%$ and $20 \%$ in stage II B. 
Table 3: Management protocols for advanced stage of cancer cervix.

\begin{tabular}{|llllll|}
\hline Treatment & IIB & IIIA & IIIIB & IVA & Total \\
\hline RT & 8 & 11 & 25 & 6 & 50 \\
\hline CT+RT & 12 & 10 & 25 & 3 & 50 \\
\hline
\end{tabular}

Table 3 out of 100 patients, 50 received combined radiotherapy and chemotherapy, and in rest of the 50 cases only radiotherapy was given.

In our study, complications observed were more with combined chemotherapy and radiotherapy than with radiotherapy alone. Out of which, vomiting was the most common complication, comprising $86 \%$ in combined therapy and $36 \%$ in radiotherapy alone. Acute toxicity of chemo radiotherapy is of short duration and resolved with medical management. Urinary complications and fistulas were not significant.

Table 4: Complications during radiotherapy and chemotherapy.

\begin{tabular}{|lll|}
\hline Complications & CT+RT \% & RT alone\% \\
\hline Vomiting & 86 & 36 \\
\hline Diarrhea & 80 & 10 \\
\hline Abdominal pain & 40 & 12 \\
\hline UTI & 20 & 14 \\
\hline CRF & 8 & 4 \\
\hline RVF & 1 & 0 \\
\hline VVF & 2 & 1 \\
\hline
\end{tabular}

Chi square $=9.1 ; \mathrm{df}=2 ; \mathrm{p}<0.05$ statistically significant

Table 5: Loco regional response.

\begin{tabular}{|lllllllll|}
\hline Loco regional response \% & IIIB & \multicolumn{10}{l|l|l|}{} & & IIIB & & IVA & (VA \\
& CT+RT & RT & CT+RT & RT & CT+RT & RT & CT+RT & RT \\
\hline Complete response & 16 & 10 & 12 & 6 & 18 & 16 & - & - \\
\hline Partial response & 8 & 4 & 6 & 16 & 26 & 22 & 2 & 2 \\
\hline No response & - & 2 & 2 & - & - & 2 & 2 & 6 \\
\hline Progressive disease & - & - & - & - & 6 & 10 & 2 & 4 \\
\hline
\end{tabular}

Chi-square $=19.59 ; \mathrm{df}=3 ; \mathrm{P}<.001$ Highly significant

Table 6: Late complications.

\begin{tabular}{|lll|}
\hline Symptoms & RT alone (\%) & CT+RT (\%) \\
\hline Skin changes & 32 & 76 \\
\hline Abdominal pain & 14 & 26 \\
\hline Diarrhea & 9 & 40 \\
\hline UTI & 20 & 34 \\
\hline CRF & 12 & 16 \\
\hline VVF & 4 & 8 \\
\hline RVF & 2 & 4 \\
\hline Vaginal stenosis & 2 & 4 \\
\hline
\end{tabular}

Chi-square $=5.54 ; \mathrm{df}=1 ; \mathrm{P}<0.05$ significant

Based on clinical examination, cytology and imaging (USG, CT Scan) loco regional response has been assessed.

In our study, in study group $16 \%$ showed complete response and $8 \%$ showed partial response in stage II B, $12 \%$ complete response, $6 \%$ partial response in stage III A, $18 \%$ complete response and $26 \%$ partial response in stage III B, in stage IV A $2 \%$ Partial response, $2 \%$ no response, $2 \%$ progressive disease.

In control group, $10 \%$ and $4 \%$ showed complete and partial response in stage II B respectively, $6 \%$ and $16 \%$ showed complete response and partial response in stage
III A respectively, in stage III B $16 \%$ showed complete response $22 \%$ partial response and $10 \%$ showed progressive disease, in stage IV A 6\% showed no response and $4 \%$ showed progressive disease.

During follow up, in study group maximum number of patients about $76 \%$ presented with skin changes like dryness, erythema, discoloration, diarrhea in $40 \%$, obstructive uropathy in $16 \%$, and in $4 \%$ developed vaginal stenosis.

In control group, 32\% had skin changes $30 \%$ with abdominal pain, $9 \%$ presented with diarrhea, obstructive uropathy in $12 \%$, vesico vaginal fistula in $4 \%$,vaginal stenosis in $2 \%$.

\section{DISCUSSION}

Data from cancer registries in developing countries indicates that more than $80-90 \%$ of cervical cancer cases develop in women 35 years or more. ${ }^{7}$ A study reported that the average age with invasive cancer was 48 years. ${ }^{8}$

We found that all patients were from the age group of 30 -60 years in the study group who received combined chemo radiotherapy and 26 patients in the control group were between the age group 61-80 years (chi-square = $8.26 \mathrm{df}=2 \mathrm{p}<.05)$ 
Study from Roswell Park, New York subsequently Kjorstad noted that in Norway during 1950 and 1960s the proportion of those less than 35 years old was only $7 \%$. Hence age itself is an independent risk factor. ${ }^{9}$ In our study $20 \%, 21 \%, 50 \%$ and $9 \%$ were in stages II B, III A, III B, IV A respectively. Maximum patients in our study were in stage III B.

The most common complications related to combined treatment are GIT related like vomiting in $86 \%$, diarrhea in $80 \%$, abdominal pain in $40 \%$ and following radiotherapy alone vomiting in $35 \%$, abdominal pain in $12 \%$, diarrhea in $10 \%$. However the complications are more common with combined therapy than with radiotherapy alone which is statistically significant (chi square $=9.1, \mathrm{df}=2 \mathrm{p}<0.05$ ) but the other complications like urinary tract infection, fistulas are not statistically significant. Also hematological complications like neutropenia are seen more with combination chemo radiotherapy.

Injury to gastrointestinal tract in 54\% usually appears within the first 2 years after radiation therapy, whereas complications of the urinary tract are seen more frequently 3 to 5 years after treatment. ${ }^{10}$

Based on the aforementioned study results, using cisplatin-based chemotherapy in combination with radiation for patients with locally advanced cervical cancer represents the standard of care.

The most common complications related to radiotherapy and chemotherapy in our study occurred in the following frequency the vomiting being the commonest in $86 \%$, followed by diarrhea in $80 \%$, abdominal pain in $40 \%$, UTI in $20 \%, \mathrm{CRF}$ in $8 \%$, vesicovaginal fistula in $2 \%$, rectovaginal fistula in 1 patient. Following radiotherapy $36 \%$ develop vomiting $10 \%$ with diarrhea $12 \%$ with abdominal pain. Hence gastrointestinal complications are more common with combined chemo radiotherapy over radiotherapy alone which is statistically significant (Chi square $=9.1, \mathrm{df}=2 \mathrm{p}<0.05$ statistically significant ).

Combined chemotherapy seems to improve the therapeutic ratio. The acute toxicity of chemo radiotherapy includes leukopenia and gastrointestinal side effects these are of short duration and resolve with medical management, while late complications of radiotherapy lead to damage which can be difficult to reverse. Combined chemo radiotherapy has become the current standard of care and in advanced disease the evidence for chemoradiation is convincing.

Kottmier HL found an incidence of bladder complications of $8 \%$ and rectal complications of $12 \% .^{11}$

\section{CONCLUSION}

As most of the cases presents in advanced stages such as stage III and IV, in which surgery is not possible, radiotherapy plays an important role in these patients. Concomitant Chemotherapy and Radiotherapy improves overall and progression-free survival rate and reduces local and distant recurrence in selected patients, which may give a cytotoxic and sensitization effect.

\section{Funding: No funding sources}

Conflict of interest: None declared

Ethical approval: The study was approved by the Institutional Ethics Committee

\section{REFERENCES}

1. Saibishkumar EP, Patel FD, Sharma SC. Evaluation of late toxicities of patients with carcinoma of the cervix treated with radical radiotherapy: An audit from India. Clin Oncol (R Coll Radiol). 2006;18:30-7.

2. Pisani P, Parkin DM, Bray, Ferlay T. Estimates of worldwide mortality from 25 Cancers in 1990. Int J Cancer. 1999;83:18-29.

3. Laara P. Changing Trends in epidemiology of Carcinoma cervix. Contribution to Obstetric Gynec. 1988;5:235.

4. Bloomfield GW. The treatment of cancer uterine cervix by radium $\mathrm{x}$-ray therapy. $\mathrm{Br} \mathrm{J}$ of Radiology. 1961;34:755.

5. Nag S, Erickson B, Thoadson B, Orlon C Damanes J D, Petereit D. the American Brachytherapy society recommendation for high dose rate brachy for ca cx. Int J Radiat Oncol Biol Physol. 2000:48:201-11.

6. Parkin DM, Bray, Plsani P, Ferlay J. Estimating the world cancer burden: Globocon 2000. Int J Cancer. 2001;94:153-6.

7. Morley GW, Lindeaure SM, Pelvic exenterative therapy for Gynecologic malignancy an analysis of 70 cases. Cancer. 1976;38(1):581-6.

8. Byckley CH, Beard CS, Fox H. Pathologic prognostic indicators in cervical cancer with particular reference to age under 40 years. Br J .of Obstetrics and Gynecology. 1988;95:47-56.

9. Kjorstad K. Carcinoma of the cervix in young patients Obstet and Gynecol. 1977;50(1):28-30.

10. Perez CA, Grigsby PW. Adjuvant chemotherapy and irradiation in locally advanced squamous cell carcinoma of the uterine cervix. PPGO Updates. 1993;1(4):1-20

11. Kottmeir HL. Complications following radiation therapy of carcinoma of cervix and their treatment. Am J Obstet and Gynecol. 1964;88:854.

Cite this article as: Rajeshwari BV. Evaluation of the effects of radiotherapy as a mode of treatment in management of advanced cancer cervix. Int J Reprod Contracept Obstet Gynecol 2017;6:379-82. 